\title{
Predicting Response to Total Neoadjuvant Treatment (TNT) in Locally Advanced Rectal Cancer Based on Multiparametric Magnetic Resonance Imaging: A Retrospective Study
}

\author{
Ganlu Ouyang (1D' \\ Xibiao Yang ${ }^{2}$ \\ Xiangbing Deng ${ }^{3}$ \\ Wenjian Meng ${ }^{3}$ \\ Yongyang $\mathrm{Yu}^{3}$ \\ Bing $\mathrm{Wu}^{2}$ \\ Dan Jiang ${ }^{4}$ \\ Pei Shu' \\ Ziqiang Wang ${ }^{3}$ \\ Jin $\mathrm{Yao}^{2}$ \\ Xin Wang'
}

'Department of Radiation Oncology/ Abdominal Oncology, Cancer Center, West China Hospital, Sichuan University, Chengdu, 61004I, People's Republic of China; ${ }^{2}$ Department of Radiology, West China Hospital, Sichuan University, Chengdu, 6I004I, People's Republic of China; ${ }^{3}$ Department of Gastrointestinal Surgery, West China Hospital, Sichuan University, Chengdu, 61004I, People's Republic of China; ${ }^{4}$ Department of Pathology, West China Hospital, Sichuan University, Chengdu, 6I004I, People's Republic of China
Correspondence: Xin Wang Department of Radiation Oncology/ Abdominal Oncology, Cancer Center West China Hospital, Sichuan University, No. 37 of Wainan Guoxue Lane, Wuhou District, Chengdu, 61004I, People's

Republic of China

Tel +86 l898060229l

Email wangxin213@sina.com
Purpose: To investigate the potential value of magnetic resonance imaging (MRI) in predicting response relevance to total neoadjuvant treatment (TNT) in locally advanced rectal cancer.

Methods: We analyzed MRI of 71 patients underwent TNT from 2015 to 2017 retrospectively. We categorized the response of TNT as CR (complete response) vs non-CR, and high vs moderate vs low sensitivity. Logistic regression analysis was used to identify the best predictors of response. Diagnostic performance was assessed using receiver operating characteristic curve analysis.

Results: Post-ICT (induction chemotherapy) $\triangle \mathrm{TL}$ (tumor length), post-CRT (concurrent chemoradiotherapy) $\triangle \mathrm{LNN}$ (the numbers of lymph node metastases), post-CCT (consolidation chemotherapy) $\Delta \mathrm{S}_{\mathrm{DWI}}$ (maximum cross-sectional area of tumor on diffusion-weighted imaging), post-CCT $\mathrm{ADC}_{\mathrm{T}}$ (the mean apparent diffusion coefficient values of tumor) and post-CCT $\triangle \mathrm{LNV}$ (volume of lymph node) were the best CR predictors. Post-ICT $\Delta \mathrm{TL}$, postCRT EMVI (extramural vascular invasion) and post-CCT $\Delta \mathrm{S}_{\mathrm{T} 2}$ (S on T2-weight) were the best significant factors for high sensitivity.

Conclusion: Post-ICT $\triangle T L$ may be an early predictor of CR and high sensitivity to TNT. Dynamic analysis based on MRI between baseline and post-CCT could provide the most valuable prediction of $\mathrm{CR}$. The grouping modality of $\mathrm{CR}$ vs non-CR may be more suitable for treatment response prediction than high vs moderate vs low sensitivity.

Keywords: rectal cancer, total neoadjuvant treatment, MRI, response, TRG

\section{Introduction}

The standard treatment for patients with locally advanced rectal cancer (LARC) is neoadjuvant concurrent chemoradiotherapy, followed by surgical resection with total mesorectal excision (TME). ${ }^{1}$ In fact, these patients have significant heterogeneity. Besides, the standard neoadjuvant therapy provided no better the overall survival (OS) and the disease-free survival (DFS) rates compared with surgery and adjuvant chemoradiotherapy. ${ }^{2}$ It is inappropriate to adopt the same therapeutic modality for all LARC patients. Recently, the National Comprehensive Cancer Network (NCCN) recommended total neoadjuvant treatment (TNT), which is an extensive and optimized therapeutic modality, as an acceptable treatment strategy for LARC. In the Phase II clinical trial of $\mathrm{TNT}^{3}$ patients received induction 
chemotherapy (ICT) followed by neoadjuvant concurrent chemoradiotherapy (CRT). After that, consolidation chemotherapy (CCT) were delivered. Some studies ${ }^{2-5}$ demonstrated that TNT might improve the pathological complete response (pCR) rate and clinical complete response (cCR) rate ranging from $14 \%$ to $36 \%$ in the patients with LARC. However, there are still some patients who have poor sensitivity to neoadjuvant chemoradiotherapy. ${ }^{6,7}$ It is of great significance to predict the response before or during treatment since the process of TNT is time-consuming. With the response predicted results, we would provide more precise and personalized treatment for patients. If the patients have good response to TNT and achieve cCR, Wait \& See strategy might be implemented. While those have not good response, we would implement other precision treatment programs that are more suitable for patients. We could establish the different treatment strategies according to tumor response.

Magnetic resonance imaging (MRI) is an important method for accurate staging and evaluation of efficacy routinely. It provides parameters to reflect the characteristics of tumors. Some studies reported that MRI parameters such as $\mathrm{T} 2$ tumor volume change, relative $\mathrm{T} 2$ signal intensity, standardized index of shape, diffusion-weighted imaging (DWI), apparent diffusion coefficient (ADC) values, tumor volume, decreased lymph node sizes and extramural vascular invasion (EMVI) may be related to predicting response. ${ }^{8-12}$ However, the conclusions of various studies were inconsistent. There is still a lack of optimal MRI prediction parameters. Additionally, TNT is a new optimization strategy for LARC. There are few relevant studies that exploring the correlation between MRI parameters and response of TNT so far. Therefore, we need to make further exploration in order to provide new evidence for precision treatment and accurate prediction of the response to neoadjuvant CRT, especially to TNT. The objective of this study was to investigate the potential value of MRI in predicting response relevance to TNT in LARC.

\section{Materials and Methods}

\section{Patient Selection}

This is a retrospective study. And the study was approved by the Ethics Committee on Biomedical Research, West China Hospital of Sichuan University (2020-903).

Patients with LARC who received TNT at our institution were retrospectively analyzed, including the patients who were enrolled in the phase II trial of TNT (ChiCTR-
OIN-17012284). ${ }^{3}$ The inclusive criteria were as follows. 1) Patients had histopathologically confirmed rectal cancer and diagnosed with stage II-III rectal cancer on MRI. 2) Patients must have completed TNT. 3) MRI scans were performed before TNT (baseline), after the end of ICT (post-ICT), CRT (post-CRT) and after CCT (post-CCT), respectively. Patients must have at least two MRI images including $\mathrm{MRI}_{\text {baseline. }}$.

\section{TNT Strategy}

\section{Chemotherapy}

One to three cycles of induction CAPOX were followed by pelvic intensity-modulated radiotherapy (IMRT)/volumetric modulated arc therapy (VMAT) and two cycles of concurrent CAPOX. Then, three cycles of consolidation CAPOX were delivered after radiotherapy. Induction and consolidation CAPOX consisted of oxaliplatin $130 \mathrm{mg} / \mathrm{m}^{2}$ on day 1 , capecitabine $1000 \mathrm{mg} / \mathrm{m}^{2}$ twice a day on day 114 , every 3 weeks. Concurrent CAPOX consisted of oxaliplatin $130 \mathrm{mg} / \mathrm{m}^{2}$ on day 1 and 22, capecitabine $825 \mathrm{mg} /$ $\mathrm{m}^{2}$ twice a day, 5 days per week for 25-28 days. Patients who received neoadjuvant pelvic radiotherapy and at least 4 cycles of neoadjuvant chemotherapy could be included in this study. No adjuvant treatment was allowed after surgery in this study.

\section{Radiotherapy}

In order to decrease the geometric uncertainty of the clinical target volume (CTV), patients were instructed to empty their bladder and then drink $500 \mathrm{~mL}$ of water 1 hour before CT simulation and each treatment, and were also instructed to move their bowels and to have an empty rectum every day. All patients were treated in the supine or prone position and immobilized with abdominal body thermoplastic masks. CT simulation was performed using a helical CT scan at $3 \mathrm{~mm}$ slice thickness, with intravenous contrast. Scanning range: The upper edge including the L2 vertebra lower edge, the lower edge is the middle femoral lower edge. The gross tumor volume (GTV) was including primary tumor and positive lymph nodes. Primary tumor, mesorectal lymph nodes, presacral lymph nodes, internal iliac lymph nodes and obturator lymph nodes were included in CTV. The planning target volume (PTV) was created by extending the CTV using a margin of $0.7-1 \mathrm{~cm}$. Radiation techniques used for the patients were IMRT or VMAT. Radiation dose to planning target volume (PTV) is $50 \mathrm{~Gy} / 25 \mathrm{f}$ or $50.4 \mathrm{~Gy} / 28 \mathrm{f}$. 


\section{Surgery}

Surgery was performed 2-3 weeks after consolidation chemotherapy. Surgery should be done according to the TME principles. All patients were recommended to receive TME. If patients reached $\mathrm{cCR}$ and rejected surgery, watch and see approach would be conducted.

\section{MR Technique}

All MR imaging were performed at a 3T Magnetom Skyra MR scanner (Siemens Healthcare). A standard T2weighted imaging (T2WI) was required by turbo spinecho in sagittal, oblique coronal (parallel to the rectum) planes, and oblique axial (perpendicular to the rectum) ${ }^{13}$, (Supplementary Figure 1). The oblique axial acquisition was performed using the following parameters: Repetition time (TR)/echo time (TE), 6890/100; Field of view (FOV), $236 \times 260 \mathrm{~mm}$; matrix, $313 \times 384$; and slice thickness $=$ $3 \mathrm{~mm}$. We used the multishot echo planar imaging (EPI) performed with a reduced TE and encoding time for DWI (Supplementary Figure 2). TR/TE, 5500/61; slice thickness $=4.5 \mathrm{~mm}$; slice gap $=0.5 \mathrm{~mm}$; FOV, $216 \times 216 \mathrm{~mm}$; matrix, $128 \times 128$; b values of 0,600 , and $1000 \mathrm{~s} / \mathrm{mm} 2$; echo spacing $=0.4 \mathrm{~ms}$; number of readout segments $=3$. The ADC map was automatically generated during image reconstruction (DWI: b values of 0 and $1000 \mathrm{~s} / \mathrm{mm} 2$ ) (Supplementary Figure 3).

\section{Data Collection}

We reviewed and collected MRI parameters, including distance of tumor (DIS), tumor length (TL), mesorectal fascia (MRF), EMVI, anal canal invasion (A), tumor stage ( $\mathrm{T}$ stage), the mean apparent diffusion coefficient values of tumor $\left(\mathrm{ADC}_{\mathrm{T}}\right), \mathrm{T} 2$ adjusted values of tumor $(\mathrm{T} 2 \mathrm{a})$, maximum cross-sectional area of tumor on diffusion-weighted imaging $\left(\mathrm{S}_{\mathrm{DWI}}\right)$ and $\mathrm{T} 2$-weight $\left(\mathrm{S}_{\mathrm{T} 2}\right)$, tumor thickness on DWI ( $\left.\mathrm{TT}_{\mathrm{DWI}}\right)$ and $\mathrm{T} 2$-weight $\left(\mathrm{TT}_{\mathrm{T} 2}\right)$, tumor volume on $\mathrm{T} 2$ weight (TV), the numbers of lymph node metastases (LNN), the longest diameter of the biggest lymph node (LND), the mean $\mathrm{ADC}$ values of the biggest lymph node $\left(\mathrm{ADC}_{\mathrm{LN}}\right)$, and volume of all lymph nodes on T2-weight (LNV), which were measured together by two experienced readers (two radiologists experience over ten years). If two readers have disagreement with each other, the discussion method will be adopted, and we recorded their consensus conclusion of the results. And the changes in MRI parameters relative to baseline were calculated including percentage change in some parameters between baseline and post-ICT/CRT/CCT MRI
$\left(\Delta \mathrm{DIS}, \Delta \mathrm{TL}, \Delta \mathrm{ADC}_{\mathrm{T}}, \Delta \mathrm{T} 2 \mathrm{a}, \Delta \mathrm{S}_{\mathrm{DWI}}, \Delta \mathrm{S}_{\mathrm{T} 2}, \Delta \mathrm{TT}_{\mathrm{DWI}}, \Delta \mathrm{TT}_{\mathrm{T} 2}\right.$, $\Delta \mathrm{TV}, \Delta \mathrm{LNN}, \Delta \mathrm{LND}, \Delta \mathrm{ADC}_{\mathrm{LN}}$ and $\Delta \mathrm{LNV}$ ), the downstage in other MRI parameters between baseline and post-ICT /CRT/CCT MRI ( $\mathrm{D}_{\mathrm{MRF}}, \mathrm{D}_{\mathrm{EMVI}}, \mathrm{D}_{\mathrm{A}}$ and $\mathrm{D}_{\mathrm{T}}$ stage $)$ (Supplementary File). The DIS that was defined as the distance from the anal verge to the inferior border of the tumor measured on sagittal T2WI. The TL was the distance from the upper edge of the tumor to the lower edge of the tumor on sagittal T2WI. Maximum cross-sectional area of tumor was considered as the product of largest diameters and its perpendicular diameters. EMVI was scored according to Smith et $\mathrm{al}^{14}$ The EMVI status was determined by the pattern of tumor margin, the size of vessel, the location of vessel relative to the tumor, and caliber of vessel. EMVI 0-2 were defined as negative, and EMVI 3 and 4 were defined as positive. We referred to Chand et al's suggestion to differentiate the post-CRT and post-CCT EMVI and fibrous bands. ${ }^{15}$ A positive lymph node was defined according to irregular borders, heterogeneous signal intensity, and round shape by Horvat et al. ${ }^{13}$ LNN was considered as the longest diameter of the biggest lymph node on oblique axial MRI scan. A maximum cross-sectional slice of tumor/lymph node was chosen as ROI which entire range delineated was used to measure the ADC value three times, and calculate the mean ADC value. ${ }^{16}$ When tumor was not observed after treatment, we will set the tumor and lymph node parameters (such as number of lymph node, length and diameter) to zero. And the ROIs were drawn on the slices of rectal wall at the same site of the previous tumor on T2WI and ADC maps. ${ }^{17} \mathrm{TV}$ and LNV were delineated on every axial slice of the CT scans, then the volume was automatically calculated by the radiotherapy planning system (pinnacle software). One radiation physician (experience 5 years) outlined ROIs and another radiation physician (experience over ten years) reviewed them. $\mathrm{T} 2 \mathrm{a}=\mathrm{T} 2$ values of tumor/T2 values of gluteus maximus muscle.

\section{Response Evaluation}

We categorized the response of TNT as complete response (CR) group vs non-CR group, and high sensitive group vs moderate sensitive group vs low sensitive group.

The pCR was defined as ypT0N0. The pCR and patients sustained $\mathrm{cCR}$ for 12 months or longer while under non-operative surveillance represented $\mathrm{CR},{ }^{4}$ whereas the rest were non-CR.

The high sensitive group (H group) included tumor regression grade (TRG) 0 and TRG 1. The moderate sensitive group (M group) defined as ether TRG 2 or 
patients with TRG 3 and tumor volume of MRI decreased by at least $20 \%$ from baseline. ${ }^{11,18}$ The low sensitive group (L group) were considered as TRG 3 and tumor volume of MRI did not decrease by $20 \%$ from baseline. The system used for TRG as recommended by the AJCC cancer Staging Manual, 8th Edition and the CAP Guidelines is that as modified from Ryan et $\mathrm{al}^{19}$ It defined TRG 0, 1, 2, 3 as no remaining viable cancer cells, only small clusters or single cancer cells remaining, residual cancer remaining but with predominant fibrosis, minimal or no tumor kill in the primary lesion but regardless of lymph node status.

\section{Statistical Analysis}

Continuous variables were expressed as mean \pm standard deviation and median (range). Categorical variables were expressed as a number (percentage). DIS, TL, $\mathrm{ADC}_{\mathrm{T}}, \mathrm{T} 2 \mathrm{a}$, $\mathrm{S}_{\mathrm{DWI}}, \mathrm{S}_{\mathrm{T} 2}, \mathrm{TT}_{\mathrm{DWI}}, \mathrm{TT}_{\mathrm{T} 2}, \mathrm{LNN}, \mathrm{LND}, \mathrm{TV}, \mathrm{LNV}, \mathrm{ADC}_{\mathrm{LN}}$ and the percentage changes in MRI parameters between baseline and post-ICT/CRT/CCT MRI were compared between independent response subgroups using Wilcoxon's rank sum test. MRF, EMVI, A, T stage, and the downstage in other MRI parameters between baseline and post-ICT/CRT /CCT MRI between independent response groups were compared using Fisher's Exact test. Logistic regression analysis was used to identify the best predictors of response. Diagnostic performance of the best predictors were assessed using receiver-operating characteristic (ROC) curve analysis, and the sensitivity, specificity, the optimal cut-off, negative predictive value (NPV), and positive predictive value (PPV) was calculated. The significance level was $\mathrm{P}<0.05$. Statistical analysis was conducted using PASW Statistics (version 25; SPSS, IBM Corp).

\section{Results}

\section{Patients}

Patients with LARC who underwent TNT before operation in our hospital from 2015 to 2017 were included. Table 1 and Supplementary Table 1-3 demonstrated characteristics of patients. The search yielded 71 patients who had baseline MR images. Median cycles of ICT, CRT and CCT were 2 cycles (range 0-5 cycles), 1 cycle (range 1-3 cycles) and 3 cycles (range $0-5$ cycles), respectively. The majority of these patients had $\mathrm{MRI}_{\mathrm{CRT}}$ and $\mathrm{MRI}_{\mathrm{CCT}}$ (55 (77.46\%) and 49 (69.02\%), respectively), 15 (21.13\%) had MRI $_{\text {ICT. There were }} 24$ patients $(33.80 \%)$ who achieved CR. In those CR patients, 23 (32.39\%) underwent surgery. Six $(8.45 \%)$ patients without cCR refused surgery after
Table I Patient Characteristics

\begin{tabular}{|c|c|}
\hline Variable & Numbers \\
\hline \multicolumn{2}{|l|}{ Cycles of chemotherapy } \\
\hline $\mathrm{ICT}$ & $2(0-5)$ cycles \\
\hline CRT & I (I-3) cycles \\
\hline CCT & $3(0-5)$ cycles \\
\hline \multicolumn{2}{|l|}{ Clinical T classification } \\
\hline $\mathrm{T} 2$ & $2(2.82 \%)$ \\
\hline T3 & 49 (69.01\%) \\
\hline $\mathrm{T} 4 \mathrm{a}$ & 14 (19.72\%) \\
\hline $\mathrm{T} 4 \mathrm{~b}$ & $6(8.45 \%)$ \\
\hline \multicolumn{2}{|l|}{ Clinical $\mathrm{N}$ classification } \\
\hline No & $6(8.45 \%)$ \\
\hline $\mathrm{NI}$ & $2(2.82 \%)$ \\
\hline N2 & $63(88.73 \%)$ \\
\hline Operation & $64(90.14 \%)$ \\
\hline Wait \& See & 7 (9.86\%) \\
\hline \multicolumn{2}{|l|}{ урТ classification } \\
\hline T0 & $24(37.50 \%)$ \\
\hline TI & $2(3.13 \%)$ \\
\hline $\mathrm{T} 2$ & $11(17.19 \%)$ \\
\hline $\mathrm{T} 3$ & $26(40.63 \%)$ \\
\hline T4 & I (1.56\%) \\
\hline \multicolumn{2}{|l|}{ ypN classification } \\
\hline No & 47 (73.44\%) \\
\hline NI & $13(20.31 \%)$ \\
\hline N2 & $4(6.25 \%)$ \\
\hline \multicolumn{2}{|l|}{ MRI } \\
\hline$M R I_{\text {baseline }}$ & 71 (100\%) \\
\hline$M R I_{\text {baseline }}+M R I_{I C T}$ & $15(21.13 \%)$ \\
\hline$M R I_{\text {baseline }}+\mathrm{MRI}_{\mathrm{CRT}}$ & 55 (77.46\%) \\
\hline$M R I_{\text {baseline }}+M R I_{C C T}$ & 49 (69.02\%) \\
\hline \multicolumn{2}{|l|}{ Response } \\
\hline $\mathrm{pCR}$ & $23(32.39 \%)$ \\
\hline cCR & I (I.4l\%) \\
\hline Non-pCR & $4 \mathrm{l}(57.75 \%)$ \\
\hline Non-cCR & $6(8.45 \%)$ \\
\hline \multicolumn{2}{|l|}{ TRG } \\
\hline 0 & $24(37.50 \%)$ \\
\hline 1 & $14(21.88 \%)$ \\
\hline 2 & $16(25.00 \%)$ \\
\hline 3 & $10(15.63 \%)$ \\
\hline \multicolumn{2}{|l|}{ Sensitivity } \\
\hline $\mathrm{H}$ group & 38 (59.38\%) \\
\hline$M$ group & $26(40.62 \%)$ \\
\hline L group & $0(0 \%)$ \\
\hline
\end{tabular}

Abbreviations: ICT, induction chemotherapy; CRT, concurrent chemoradiotherapy; $\mathrm{CCT}$, consolidation chemotherapy; $\mathrm{PCR}$, pathological complete response; $\mathrm{CCR}$, clinical complete response; $\mathrm{H}$ group, the high sensitive group; $\mathrm{M}$ group, the moderate sensitive group; L group, the low sensitive group. 
TNT. Only one person (1.41\%) sustained CR for 12 months and received Wait \& See. Among the 71 patients in the TNT cohort, the pathological TRG was TRG 0 in 24 (37.50\%) patients, TRG 1 in $14(21.88 \%)$, TRG 2 in 16 (25.00\%) and TRG 3 in 10 (15.63\%). According to TRG, $38(59.38 \%)$ patients were classified as $\mathrm{H}$ group, 26 (40.63\%) were classified as the $\mathrm{M}$ group, and no patients was L group. Figure 1 shows the Distribution of CR vs non-CR and $\mathrm{H}$ group vs $\mathrm{M}$ group in the $\mathrm{MRI}_{\mathrm{ICT}}, \mathrm{MRI}_{\mathrm{CRT}}$ and $\mathrm{MRI}_{\mathrm{CCT}}$. There was a patient who was TRG 0 but ypN1. Therefore, we believe that he belongs to the high sensitive group and non-pCR group.

\section{Correlation Between MRI Parameters and Response to TNT}

No significant correlation was noted between response and baseline MRI parameters. Correlations between $\mathrm{CR}$ and post-ICT $\Delta \mathrm{TL}(\mathrm{p}=0.008)$, post-ICT $\Delta \mathrm{LND}(\mathrm{p}=0.019)$ and post-ICT $\Delta \mathrm{LNV}(\mathrm{p}=0.019)$ were found. Post-ICT $\Delta \mathrm{TL}$ was also correlated with high sensitivity to TNT with the $\mathrm{p}$ value 0.01 (Table 2).

There were significant correlations between CR and post-CRT EMVI ( $\mathrm{p}=0.002)$, post-CRT LNN ( $\mathrm{p}=$ 0.004), post-CRT LND ( $\mathrm{p}=0.021)$, post-CRT $\Delta \mathrm{LNN}$ $(\mathrm{p}=0.002)$ and post-CRT $\Delta \mathrm{LND}(\mathrm{p}=0.021)$. Only postCRT EMVI was correlated with high sensitivity of LARC to TNT ( $\mathrm{p}=0.013$ ) (Table 3$)$.

The following parameters of post-CCT MRI which were significant correlations between $\mathrm{CR}$ were evaluated: post-CCT $\mathrm{ADC}_{\mathrm{T}}(\mathrm{p}=0.008)$, post-CCT TT $\mathrm{TWI}(\mathrm{p}=0.031)$, post-CCT LNV ( $p=0.003)$, post-CCT LNN $(p=0.016)$, post-CCT $\Delta \mathrm{S}_{\mathrm{DWI}}(\mathrm{p}=0.001)$, post-CCT $\Delta \mathrm{S}_{\mathrm{T} 2}(\mathrm{p}=0.006)$, post-CCT $\Delta \mathrm{TT}_{\mathrm{DWI}}(\mathrm{p}=0.029)$, post-CCT $\Delta \mathrm{LNN}(\mathrm{p}=0.008)$,
post-CCT $\triangle \mathrm{LND}(\mathrm{p}=0.046)$ and post-CCT $\triangle \mathrm{LNV}(\mathrm{p}=$ 0.002). There were many post-CCT MRI parameters had significant correlations between high sensitivity to TNT: post-CCT TL $(p=0.005)$, post-CCT $\mathrm{S}_{\mathrm{DWI}}(\mathrm{p}=0.036)$, post$\operatorname{CCT~S}_{\mathrm{T} 2}(\mathrm{p}=0.008)$, post-CCT TT $\mathrm{DWI}(\mathrm{p}=0.037)$, post-CCT $\mathrm{TT}_{\mathrm{T} 2}(\mathrm{p}=0.009)$, post-CCT LNV $(\mathrm{p}=0.044)$, post-CCT $\Delta \mathrm{TL}$ $(\mathrm{p}=0.004)$, post-CCT $\Delta \mathrm{S}_{\mathrm{DWI}}(\mathrm{p}=0.009)$, post-CCT $\Delta \mathrm{S}_{\mathrm{T} 2}(\mathrm{p}$ $=0.001)$, post-CCT $\Delta \mathrm{TT}_{\mathrm{T} 2}(\mathrm{p}=0.009)$, post-CCT $\Delta \mathrm{TV}(\mathrm{p}=$ $0.007)$, post-CCT $\Delta \mathrm{LNN}(\mathrm{p}=0.016)$, post-CCT $\Delta \mathrm{LNV}(\mathrm{p}=$ $0.019)$ and post-CCT $\mathrm{D}_{\mathrm{T} \text { stage }}(\mathrm{p}=0.022)$ (Table 4$)$.

Based on the results of above analysis, ultimately we selected using the method of binary logistic regression to build the response early predicting models. In the binary logistic regression analysis, post-ICT $\triangle \mathrm{TL}(95 \% \mathrm{CI}$ : 2.606-3.208, $\mathrm{p}=0.038)$, post-CRT $\Delta \mathrm{LNN} \quad(95 \%$ CI: $1.209-80.258, \mathrm{p}=0.033)$, post-CCT $\Delta \mathrm{S}_{\mathrm{DWI}}(95 \% \mathrm{CI}$ : $6.374-40.883, \mathrm{p}=0.01)$, post-CCT $\mathrm{ADC}_{\mathrm{T}}(95 \% \mathrm{CI}:$ 27.517-52.047, $\mathrm{p}=0.003)$ and post-CCT $\triangle \mathrm{LNV}(95 \%$ CI: $35.108-61.120, p=0.017)$ were found to be the best predictors for CR. Moreover, post-ICT $\triangle \mathrm{TL}(95 \% \mathrm{CI}$ : $2.720-3.121, \quad p=0.001)$, post-CRT EMVI $(95 \%$ CI: $1.55-52.266, \mathrm{p}=0.013)$ and post-CCT $\Delta \mathrm{S}_{\mathrm{T} 2}(95 \%$ CI: $0.004-0.392, p=0.006)$ were the best significant factors for high sensitivity to TNT (Tables 2-4). Figure 5 showed post-ICT $\triangle \mathrm{TL}$ of CR vs non $-\mathrm{CR}$ and $\mathrm{H}$ group vs $\mathrm{M}$ group for each patient.

\section{Prediction Performances of MRI Parameters for Response}

Based on logistic regression model, ROC curve analysis was used to explore the role of the best predictors. Figure 2 presents ROC curve results for MRI parameters differentiating $\mathrm{CR}$ from non-CR. Besides, ROC curve
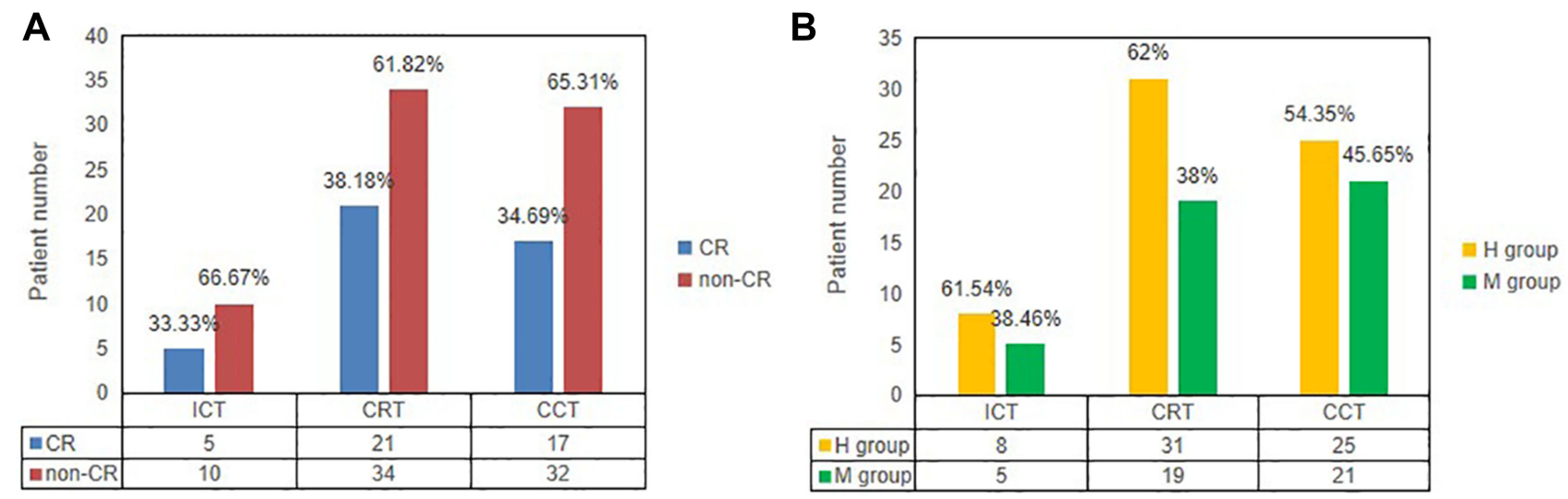

Figure I (A) Distribution of $C R$ vs non-CR in the $M R I_{I C T}$, $M R I_{C R T}$ and $M R I_{C C T}$. (B) Distribution of the high sensitive group (H group) vs the moderate sensitive group ( $M$ group) in the $\mathrm{MRI}_{\mathrm{ICT}}, \mathrm{MRI}_{\mathrm{CRT}}$ and $\mathrm{MRI}_{\mathrm{CCT}}$. 
Table 2 Logistic Regression Analysis of Post-ICT Predictive Factors of CR and High Sensitivity to TNT

\begin{tabular}{|l|l|l|l|l|l|l|}
\hline \multirow{2}{*}{ Parameters } & Univariate (CR) & \multicolumn{2}{|c|}{ Multivariate (CR) } & Univariate (H Group) & \multicolumn{2}{|c|}{ Multivariate (H Group) } \\
\cline { 2 - 7 } & P & $\mathbf{9 5 \%}$ Cl & P & P & $\mathbf{9 5 \%}$ Cl & P \\
\hline Post-ICT $\Delta$ TL & $0.008^{*}$ & $2.606-3.208$ & $0.038^{*}$ & $0.0 I^{*}$ & - & - \\
Post-ICT $\Delta$ LND & $0.019^{*}$ & & 0.988 & 0.300 & & \\
Post-ICT $\Delta$ LNV & $0.019^{*}$ & & 0.988 & 0.188 & \\
\hline
\end{tabular}

Note: $* \mathrm{P}<0.05$.

Abbreviations: ICT, induction chemotherapy; TL, tumor length; LND, diameter of lymph node; LNV, volume of lymph node on T2 - weight.

Table 3 Logistic Regression Analysis of Post-CRT Predictive Factors of CR and High Sensitivity to TNT

\begin{tabular}{|c|c|c|c|c|c|c|}
\hline \multirow[t]{2}{*}{ Parameters } & \multirow{2}{*}{$\begin{array}{l}\text { Univariate (CR) } \\
P\end{array}$} & \multicolumn{2}{|c|}{ Multivariate (CR) } & \multirow{2}{*}{$\begin{array}{l}\text { Univariate (H Group) } \\
P\end{array}$} & \multicolumn{2}{|c|}{ Multivariate (H Group) } \\
\hline & & $95 \% \mathrm{Cl}$ & $\mathbf{P}$ & & 95\% Cl & $\mathbf{P}$ \\
\hline Post-CRT EMVI & $0.002^{*}$ & & 0.709 & $0.013^{*}$ & - & - \\
\hline Post-CRT LNN & $0.004 *$ & & 0.978 & 0.125 & & \\
\hline Post-CRT LND & $0.021^{*}$ & & 0.896 & 0.272 & & \\
\hline Post-CRT $\Delta \mathrm{LNN}$ & $0.002 *$ & $1.209-80.258$ & $0.033^{*}$ & 0.252 & & \\
\hline Post-CRT $\Delta$ LND & $0.021 *$ & & 0.896 & 0.242 & & \\
\hline
\end{tabular}

Note: $* \mathrm{P}<0.05$.

Abbreviations: CRT, concurrent chemoradiotherapy; EMVI, extramural vascular invasion; LNN, the numbers of lymph node metastases; LND, diameter of lymph node.

Table 4 Logistic Regression Analysis of Post-CCT Predictive Factors of CR and High Sensitivity to TNT

\begin{tabular}{|c|c|c|c|c|c|c|}
\hline \multirow[t]{2}{*}{ Parameters } & \multirow{2}{*}{$\begin{array}{l}\text { Univariate (CR) } \\
P\end{array}$} & \multicolumn{2}{|c|}{ Multivariate (CR) } & \multirow{2}{*}{$\begin{array}{l}\text { Univariate (H Group) } \\
P\end{array}$} & \multicolumn{2}{|c|}{ Multivariate (H Group) } \\
\hline & & $95 \% \mathrm{Cl}$ & $\mathbf{P}$ & & $95 \% \mathrm{Cl}$ & $\mathbf{P}$ \\
\hline Post-CCT ADC ${ }_{T}$ & $0.008^{*}$ & $27.517-52.047$ & $0.003 *$ & 0.063 & & - \\
\hline Post-CCT TT $\mathrm{DWI}_{\mathrm{DI}}$ & $0.03 I^{*}$ & & 0.205 & $0.037^{*}$ & & 0.290 \\
\hline Post-CCT $\Delta \mathrm{TT}_{\mathrm{DW}}$ & $0.029 *$ & & 0.28 & 0.056 & & - \\
\hline Post-CCT TT $\mathrm{T}_{\mathrm{T} 2}$ & 0.08 & & - & $0.009 *$ & & 0.89 \\
\hline Post-CCT $\Delta \mathrm{TT}_{\mathrm{T} 2}$ & $0.048^{*}$ & & 0.705 & $0.009 *$ & & 0.221 \\
\hline Post-CCT S SWI & 0.064 & & - & $0.036^{*}$ & & 0.631 \\
\hline Post-CCT $\Delta \mathrm{S}_{\mathrm{DW}}$ & $0.001 *$ & $6.374-40.883$ & $0.01 *$ & $0.009 *$ & & 0.993 \\
\hline Post-CCT $\mathrm{S}_{\mathrm{T} 2}$ & 0.104 & & - & $0.008^{*}$ & & 0.933 \\
\hline Post-CCT $\Delta \mathrm{S}_{\mathrm{T} 2}$ & $0.006 *$ & & 0.058 & $0.001 *$ & $0.004-0.392$ & $0.006^{*}$ \\
\hline Post-CCT LNN & $0.016^{*}$ & & 0.998 & 0.103 & & - \\
\hline Post-CCT $\triangle \mathrm{LNN}$ & $0.008^{*}$ & & 0.127 & $0.016 *$ & & 0.209 \\
\hline Post-CCT $\Delta \mathrm{LND}$ & $0.046^{*}$ & & 0.067 & 0.056 & & - \\
\hline Post-CCT LNV & $0.003 *$ & & 0.358 & $0.044^{*}$ & & 0.607 \\
\hline Post-CCT $\Delta \mathrm{LNV}$ & $0.002 *$ & $35.108-61.120$ & $0.017 *$ & $0.019 *$ & & 0.439 \\
\hline Post-CCT $\Delta T V$ & 0.069 & & - & $0.007^{*}$ & & 0.338 \\
\hline Post-CCT TL & 0.053 & & - & $0.005^{*}$ & & 0.356 \\
\hline Post-CCT $\Delta T L$ & 0.098 & & - & $0.004^{*}$ & & 0.371 \\
\hline Post-CCT $D_{\mathrm{T} \text { stage }}$ & $0.022^{*}$ & & 0.421 & $0.022 *$ & & $0.56 \mathrm{I}$ \\
\hline
\end{tabular}

Note: $* \mathrm{P}<0.05$

Abbreviations: $C C T$, consolidation chemotherapy; $\mathrm{ADC}_{\mathrm{T}}$, the mean apparent diffusion coefficient values of tumor; $\mathrm{TT}_{\mathrm{DWl}}$, tumor thickness on $\mathrm{DWI}_{\mathrm{W}} \mathrm{TT}_{\mathrm{T} 2}$, tumor thickness on T2 - weight; $\mathrm{S}_{\mathrm{DW}}$, maximum cross - sectional area of tumor on diffusion-weighted imaging; $\mathrm{S}_{\mathrm{T} 2}$, maximum cross - sectional area of tumor on T2 - weight; $\mathrm{LNN}$, the numbers of lymph node metastases; LND, diameter of lymph node; LNV, volume of lymph node on T2 - weight; TV, tumor volume on T2 - weight; TL, tumor length; T stage, tumor stage.

results are reported that MRI parameters predicting $\mathrm{H}$ group in Figure 3. Corresponding data are provided in Table 5. In terms of individual parameter prediction response, post-ICD $\triangle \mathrm{TL}$ was selected as the best predictor of CR by logistic regression model (AUC 0.92, specificity $80 \%$, sensitivity 100\%, NPV 100\%, PPV 71.4\%, ACC 

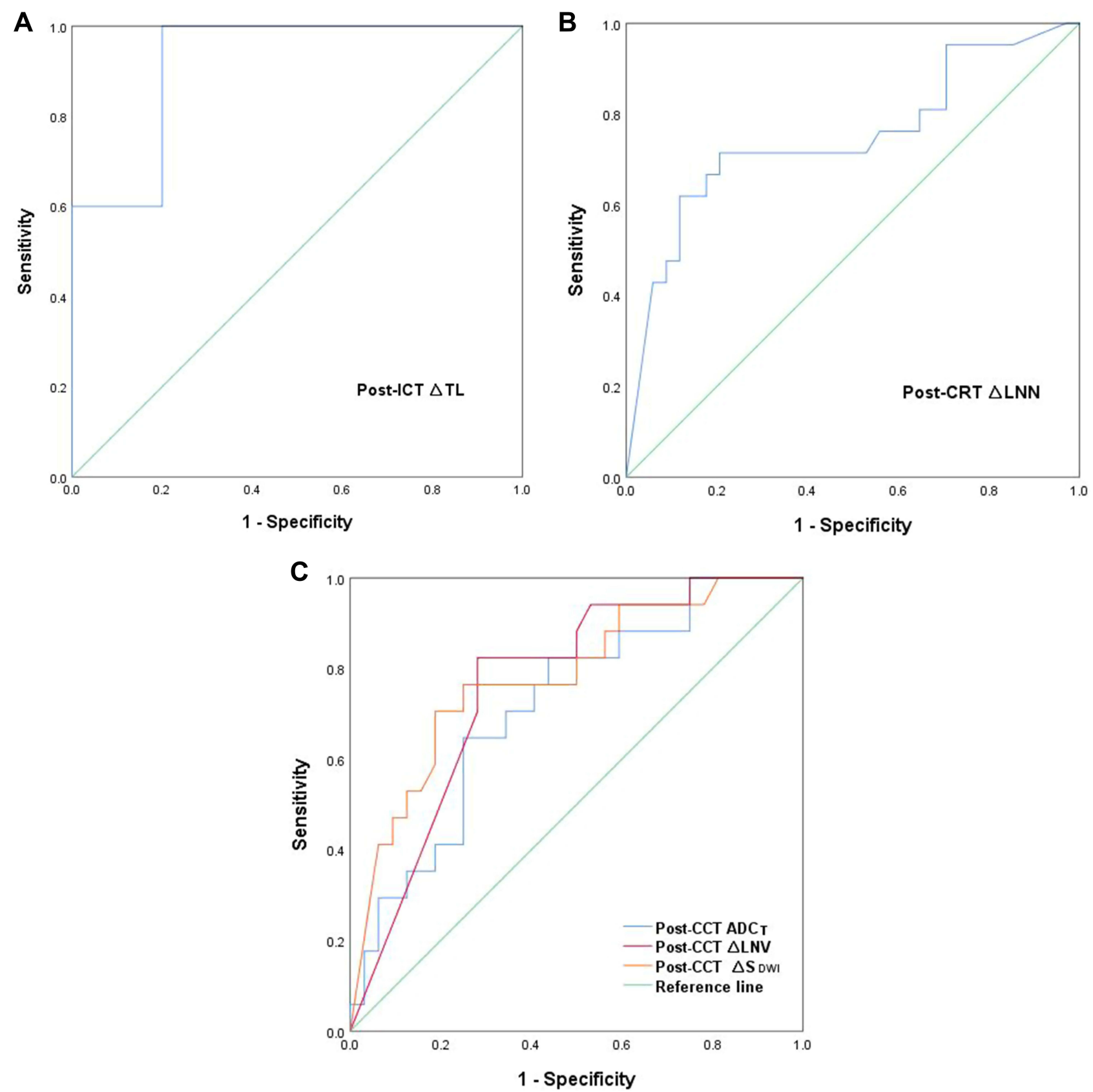

Figure 2 (A) ROC curves of predicting CR in the post-ICT MRI cohorts. (B) ROC curves of predicting CR in the post-CRT MRI cohorts. (C) ROC curves of predicting CR in the post-CCT MRI cohorts.

$86.7 \%, \mathrm{p}=0.01)$. ROC analysis also showed that post-ICT $\Delta \mathrm{TL}$ had a moderate predicting performance in identifying $\mathrm{H}$ group (AUC 0.84, specificity 100\%, sensitivity 75\%, NPV 70.4\%, PPV 96\%, ACC 82.7\%, p $=0.001)$. In particular, the combination of post-CCT $\Delta \mathrm{S}_{\mathrm{DWI}}$, postCCT LNV and post-CCT $\mathrm{ADC}_{\mathrm{T}}$ had best predicting performance of CR, with AUC 0.94, a sensitivity of $94.1 \%$, and a specificity of $90.6 \%$ (Figure 4 ).

\section{Discussion}

In the present study, MRI parameters of TNT patients in three different neoadjuvant treatment phases were used to predict response. It is the first study that using MRI parameters to predict response of TNT. Most studies have used good responder and poor responder according to TRG as an evaluation method. ${ }^{9,20,21}$ Actually, TRG is subjective. $^{22}$ The sensitivity to TNT was grouped 

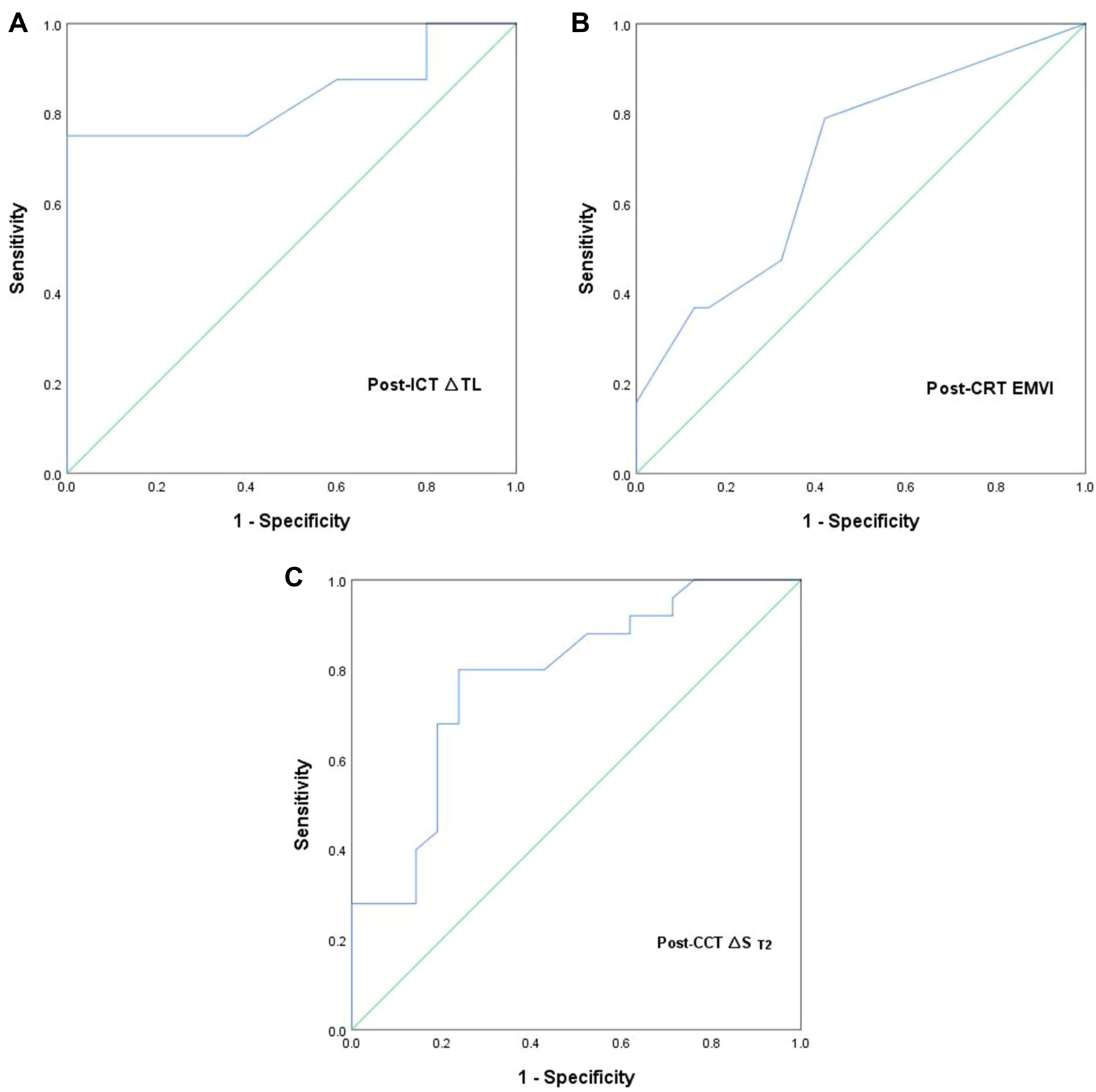

Figure 3 (A) ROC curves of predicting the high sensitive group ( $\mathrm{H}$ group) in the post-ICT MRI cohorts.(B) ROC curves of predicting the high sensitive group (H group) in the post-CRT MRI cohorts. (C) ROC curves of predicting the high sensitive group ( $\mathrm{H}$ group) in the post-CCT MRI cohorts.

according to tumor volume reduction rate on MRI and TRG of LARC following treatment with TNT. In this study, there was no $\mathrm{L}$ group but only $\mathrm{H}$ group and $\mathrm{M}$ group, which may be because TNT is the strongest neoadjuvant therapy. More studies ${ }^{3,23-25}$ have reported that patients could achieve a high rate of CR. We used two modalities to describe the response to TNT, CR vs non-CR group and high sensitive vs moderate sensitive vs low sensitive group. And our results revealed that
MRI parameters could predict CR. So the grouping modality of CR vs non-CR may be more suitable for treatment response prediction.

We found post-ICT $\Delta \mathrm{TL}$ offered the good results for the detection of patients with a CR and high sensitivity after TNT. Currently, there are very few researches that have described MRI parameters of post-ICT $\Delta \mathrm{TL}$ for predicting a $\mathrm{CR}$ and high sensitivity. The smaller post-nCRT tumor length predicted an increased pCR rate in the 

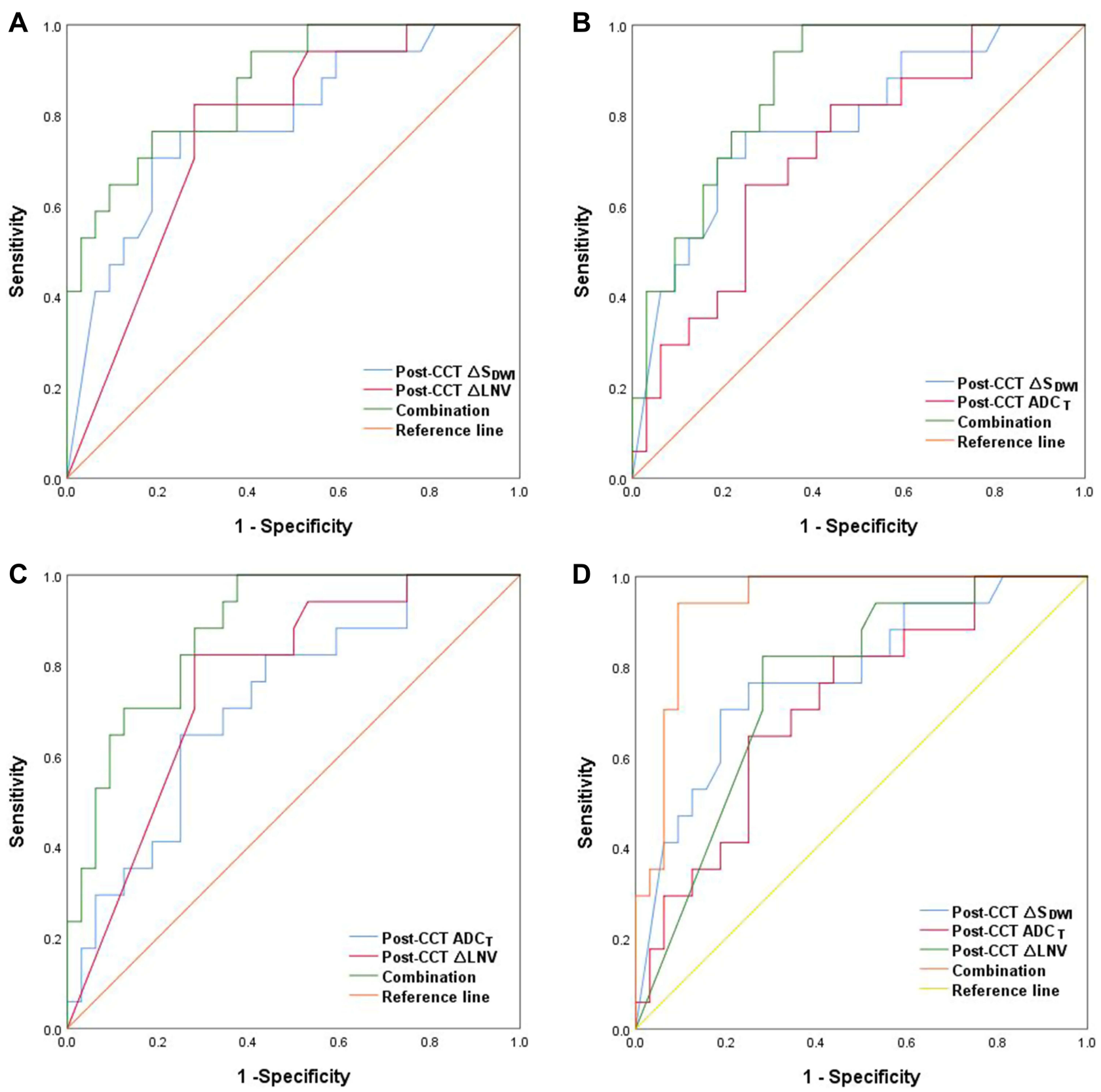

Figure 4 (A) ROC curves of predicting $C R$ (combination of post-CCT $\triangle S_{D W I}$ and post-CCT $\triangle L N V$ ). (B) ROC curves of predicting CR (combination of post-CCT $\triangle S_{D W I}$ and post-CCT ADC $\mathrm{T}$ ). (C) ROC curves of predicting $C R$ (combination of post-CCT ADC $\mathrm{T}_{T}$ and post-CCT $\triangle \mathrm{LNV}$ ). (D) ROC curves of predicting $C R$ (combination of post$C C T \Delta S_{D W l}$, post-CCT ADC $T$ and post-CCT $\Delta L N V$ ).

previous studies. ${ }^{20,26,27}$ FOWARC analyzed MRI images of 403 patients and found that baseline TL was a significant factor for predicting pCR probability and patients with TL $(>3 \mathrm{~cm})$ may have a lower pCR probability. ${ }^{28}$ In our study, there was significant difference of post-ICT $\triangle \mathrm{TL}$ in predicting $\mathrm{CR}$ and high sensitivity. Although our sample size is only 15 cases, integrating the result and practice experience we may conclude that the larger the post-ICT $\Delta \mathrm{TL}$ predicted the more the tumor regression and the higher the probability of $\mathrm{CR}$ and high sensitivity. Post-ICT $\Delta \mathrm{TL}$ might be an early prediction parameter of $\mathrm{CR}$ and high sensitivity. It has important reference value to help predict the response of neoadjuvant chemoradiotherapy and adjust the treatment plan as soon as possible.

Unlike other studies, we explored that post-CRT EMVI had a great predicting performance in identifying $\mathrm{H}$ group. When the post-CRT EMVI status was negative, sensitivity of 

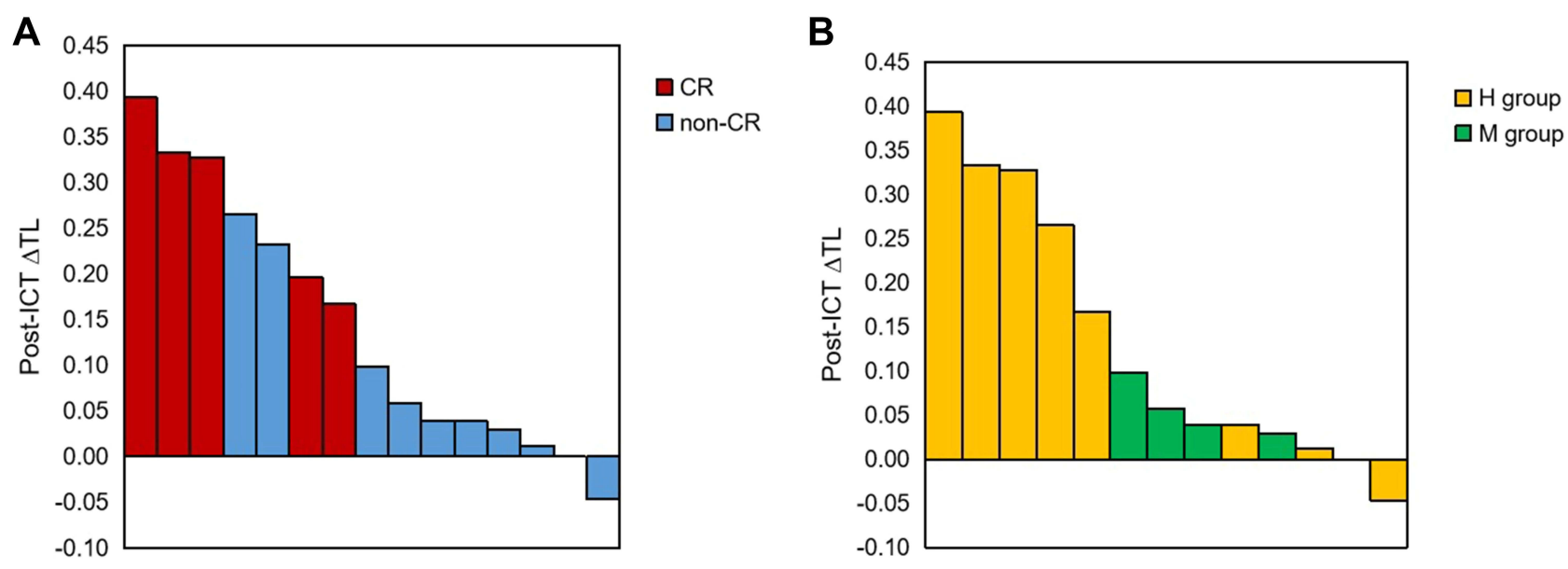

Figure 5 (A) Post - ICT $\Delta T L$ of CR vs non - CR for each patient. (B) Post - ICT $\Delta T L$ of the high sensitive group (H group) vs the moderate sensitive group (M group) for each patient.

patients to TNT was higher than positive. In previous literature by Lee et al, ${ }^{12}$ post-CRT EMVI was the only significant MRI factor in DFS. Long-term results from the GEMCAD 0801 trial $^{29}$ and Meng et $\mathrm{al}^{30}$ considered baseline mrEMVI positivity was an independent prognostic indicator for DFS. Most previous studies have explored the relationship between EMVI and prognosis, yet we explored the correlation between EMVI and response and obtained good positive results. This maybe because radiation was effective in wiping out pathways of vascular spread in the pelvis. ${ }^{29}$
Regarding the definition of lymph node, we defined the positive lymph node according to Horvat et al. ${ }^{13}$ In the study, the more post-CRT $\triangle \mathrm{LNN}$ and post-CCT $\Delta \mathrm{LNV}$ provoked more chance of $\mathrm{CR}$ and high sensitivity to TNT in our study. At this point, patients who had postCRT $\Delta \mathrm{LNN} \geq 70 \%$ would be more easier to achieve CR. Bustamante-Lopez et $\mathrm{al}^{31}$ found only $\mathrm{pCR}$ showed a significant association with $<12$ baseline LN. However, none of the previous studies had indicated correlation between post-CRT $\triangle \mathrm{LNN}$ or post-CCT $\Delta \mathrm{LNV}$ and

Table 5 Multivariate Analysis Results About Magnetic Resonance Imaging (MRI) Findings for the Prediction of CR and High Sensitivity to TNT

\begin{tabular}{|c|c|c|c|c|c|c|c|}
\hline Parameters & $\operatorname{AUC}(95 \% \mathrm{Cl})$ & SEN & SPE & PPV & NPV & ACC & $P$ value \\
\hline \multicolumn{8}{|l|}{ MRI findings for the prediction of CR } \\
\hline Post-ICD $\Delta T L$ & $0.92(0.778-1.000)$ & $100 \%$ & $80 \%$ & $71.4 \%$ & $100 \%$ & $86.7 \%$ & $0.01 *$ \\
\hline Post-CRT $\triangle \mathrm{LNN}$ & $0.75(0.603-0.891)$ & $71.4 \%$ & $79.4 \%$ & $68.2 \%$ & $81.8 \%$ & $76.4 \%$ & $0.002 *$ \\
\hline Post-CCT $\Delta \mathrm{S}_{\mathrm{DW}}$ & $0.78(0.646-0.92)$ & $70.6 \%$ & $81.2 \%$ & $66.7 \%$ & $83.9 \%$ & $77.6 \%$ & $0.001 *$ \\
\hline Post-CCT ADC & $0.72(0.672-0.866)$ & $64.7 \%$ & $75 \%$ & $57.9 \%$ & $80 \%$ & $71.4 \%$ & $0.012^{*}$ \\
\hline Post-CCT $\Delta \mathrm{LNV}$ & $0.76(0.629-0.899)$ & $82.4 \%$ & $71.9 \%$ & $60.9 \%$ & $88.5 \%$ & $75.5 \%$ & $0.003^{*}$ \\
\hline Post-CCT $\Delta S_{D W l}+$ Post-CCT $A D C_{T}$ & $0.86(0.766-0.962)$ & $94.1 \%$ & $68.7 \%$ & $61.5 \%$ & $95.7 \%$ & $77.6 \%$ & $<0.001 *$ \\
\hline Post-CCT $\Delta \mathrm{S}_{\mathrm{DWI}}+$ Post-CCT $\Delta \mathrm{LNV}$ & $0.87(0.766-0.97)$ & $76.5 \%$ & $81.2 \%$ & $68.4 \%$ & $86.7 \%$ & $79.6 \%$ & $<0.00 I^{*}$ \\
\hline Post-CCT ADC $\mathrm{T}_{\mathrm{T}}+$ Post-CCT $\Delta \mathrm{LNV}$ & $0.88(0.786-0.972)$ & $100 \%$ & $62.5 \%$ & $58.6 \%$ & $100 \%$ & $75.5 \%$ & $<0.001 *$ \\
\hline Post-CCT $\Delta \mathrm{S}_{\mathrm{DWl}}+$ Post-CCT ADC + Post-CCT $\Delta \mathrm{LNV}$ & $0.94(0.873-1)$ & $94.1 \%$ & $90.6 \%$ & $84.2 \%$ & $96.7 \%$ & $91.8 \%$ & $<0.00 I^{*}$ \\
\hline \multicolumn{8}{|l|}{ MRI findings for the prediction of high sensitivity to TNT } \\
\hline Post-CRT EMVI & $0.69(0.543-0.846)$ & $100 \%$ & $80 \%$ & $76 \%$ & $100 \%$ & $68 \%$ & $0.022^{*}$ \\
\hline Post-CCT $\Delta \mathrm{S}_{\mathrm{T} 2}$ & $0.78(0.645-0.917)$ & $80 \%$ & $76.2 \%$ & $80 \%$ & $76.2 \%$ & $78.3 \%$ & $0.001 *$ \\
\hline Post-ICD $\Delta \mathrm{TL}$ & $0.84(0.610-1.000)$ & $75 \%$ & $100 \%$ & $96 \%$ & $70.4 \%$ & $82.7 \%$ & $0.048^{*}$ \\
\hline
\end{tabular}

Note: $* \mathrm{P}<0.05$.

Abbreviations: AUC, area under curve; SEN, sensitivity; SPE, specificity; PPV, positive predictive value; NPV, negative predictive value; ACC, accuracy; MRI, magnetic resonance imaging; ICT, induction chemotherapy; CRT, concurrent chemoradiotherapy; CCT, consolidation chemotherapy; TL, tumor length; $L N N$, the numbers of lymph node metastases; $\mathrm{S}_{\mathrm{DWl}}$, maximum cross - sectional area of tumor on diffusion-weighted imaging; $A D C_{T}$, the mean apparent diffusion coefficient values of tumor; $L N V$, volume of lymph node on T2 - weight; EMVI, extramural vascular invasion; $\mathrm{S}_{\mathrm{T} 2}$, maximum cross - sectional area of tumor on T2 - weight. 
response so far. It may be associated with the definition of positive lymph node is different and subjective.

DWI is increasingly incorporated in clinical rectal MRI exams worldwide. ${ }^{32}$ Moreover, the DWI-derived ADC values can be used for quantitative analysis of tumorous cellular density and extracellular space. ${ }^{16}$ ADC values are mainly negatively related to cell density and positively related to extracellular space. ${ }^{16,20,21}$ After consolidation chemotherapy, some tumor tissue was replaced by fibrous tissue. Post-CCT $\mathrm{ADC}_{\mathrm{T}}$ reflects the tissue density after TNT rather than only the tumor cell. Fibrotic tissue generally has low ADC. ${ }^{21}$ Consequently, Post-CCT $\mathrm{ADC}_{\mathrm{T}}$ may be a useful parameter for discriminating between $\mathrm{CR}$ and non-CR. Several articles focused on post-CRT $\mathrm{ADC}$ and post-CRT $\triangle \mathrm{ADC} .^{33-35}$ However, we found $\mathrm{ADC}_{\mathrm{T}}$ of post-CCT MRI was associated with response. This differs from above studies.

Post-CCT MRI, which was performed after all neoadjuvant treatment, was mainly used for the diagnosis of cCR or pCR. We did not evaluate the accuracy of diagnosis of cCR using post-CCT MRI, and tried to avoid inconsistencies between cCR and pCR. In the study, we also performed a dynamic analysis based on baseline and post-ICT/CRT/CCT MRI. And post-CCT $\Delta \mathrm{S}_{\mathrm{DWI}}$, post$\mathrm{CCT} \mathrm{ADC}_{\mathrm{T}}$ and post-CCT $\Delta \mathrm{LNV}$ were found to be the best predictors for CR. Post-CCT $\Delta \mathrm{S}_{\mathrm{T} 2}$ were the best significant factors for high sensitivity to TNT. Moreover, the combination of post-CCT $\Delta \mathrm{S}_{\mathrm{DWI}}$, post-CCT LNV and post-CCT $\mathrm{ADC}_{\mathrm{T}}$ had best predicting performance of $\mathrm{CR}$, which improved the diagnostic performance of $\mathrm{CR}$ and treatment response comparing the single MRI parameter. Of those parameters, post-CCT $\Delta \mathrm{S}_{\mathrm{DWI}}$, post-CCT $\Delta \mathrm{LNV}$ and post-CCT $\Delta \mathrm{S}_{\mathrm{T} 2}$, calculated by parameters of baseline MRI and post-CCT MRI, were dynamic parameters. Therefore, our results revealed that dynamic analysis based on MRI between baseline and post-CCT could provide the most valuable prediction of $\mathrm{CR}$. In addition, combining the multiple MRI parameters improved the diagnostic performance of $\mathrm{CR}$ and treatment response comparing with the single MRI parameter.

There were a few limitations in the study that must be considered. Firstly, our study was a retrospective analysis with a small sample size collected from a single center, thus we can further enlarge sample size and get data from multicenter to validate the results. In addition, we did not compare the same parameter of post-ICT MRI, post-CRT MRI and post-CCT MRI to confirm that it can better reflect the response at a certain phase. Because many patients may have only two phases of treatments. Thirdly, we assessed $\mathrm{S}_{\mathrm{DWI}}$ and $\mathrm{S}_{\mathrm{T} 2}$ by measuring manually its long diameter and short diameter, and the area measured may be not accurate. Use of the image processing software may increase the accuracy.

\section{Conclusions}

Consequently, post-ICT $\Delta \mathrm{TL}$, post-CRT $\Delta \mathrm{LNN}$, post-CCT $\Delta \mathrm{S}_{\mathrm{DWI}}$, post-CCT $\mathrm{ADC}_{\mathrm{T}}$ and post-CCT $\Delta \mathrm{LNV}$ were related to CR. Post-ICT $\Delta$ TL, post-CRT EMVI and postCCT $\Delta \mathrm{S}_{\mathrm{T} 2}$ were correlated with high sensitivity to TNT. Post-ICT $\Delta \mathrm{TL}$ may be an early predictor of CR and high sensitivity. In addition, the combination of post-CCT $\Delta \mathrm{S}_{\mathrm{DWI}}$, post-CCT LNV and post-CCT $\mathrm{ADC}_{\mathrm{T}}$ had best predicting performance of $\mathrm{CR}$. Dynamic analysis based on MRI between baseline and post-CCT could provide the most valuable prediction of CR. Moreover, radiomics $^{17,36-38}$ and deep learning methods ${ }^{39-41}$ could be used as good tools for image data mining. Therefore, on the one hand, further studies based on artificial intelligence are indicated to explore the best predictor of response. On the other hand, in the future, we can enlarge sample size and get data from multicenter to validate the results.

\section{Ethical Approval}

This is a retrospective study. And the study was approved by the Ethics Committee on Biomedical Research, West China Hospital of Sichuan University (2020-903). Patient consent was not required. The reasons for the waiver were as follows. Firstly, this is a retrospective study. There was no additional risk to patients. In the process of ethical approval, we have submitted to the ethics committee the application for exemption from informed consent of patients. Additionally, we abided by the Declaration of Helsinki. We collected de-identified data of patients. And the final results of the study would be anonymity.

\section{Acknowledgments}

Supported by grants from the National Natural Science Foundation of China (82073338), grants from Science and Technology bureau of Chengdu of China (2019YF05-00771-SN), grants from the 1.3.5 Project for Disciplines of Excellence - Clinical Research Incubation Project, West China Hospital, Sichuan University (2020HXFH002). 


\section{Disclosure}

The authors report no conflicts of interest in this work.

\section{References}

1. Sauer R, Becker H, Hohenberger W, et al. Preoperative versus postoperative chemoradiotherapy for rectal cancer. $N$ Engl $J$ Med. 2004;351(17):1731-1740. doi:10.1056/NEJMoa040694

2. Petrelli F, Trevisan F, Cabiddu M, et al. Total neoadjuvant therapy in rectal cancer: a systematic review and meta-analysis of treatment outcomes. Ann Surg. 2020;271(3):440-448. doi:10.1097/SLA.00000 00000003471

3. Wang X, Yu Y, Meng W, et al. Total neoadjuvant treatment (CAPOX plus radiotherapy) for patients with locally advanced rectal cancer with high risk factors: a Phase 2 trial. Radiother Oncol. 2018;129 (2):300-305. doi:10.1016/j.radonc.2018.08.027

4. Cercek A, Roxburgh CSD, Strombom P, et al. Adoption of total neoadjuvant therapy for locally advanced rectal cancer. JAMA Oncol. 2018;4(6):e180071. doi:10.1001/jamaoncol.2018.0 071

5. Hu YH, Wei JW, Chang H, et al. The high pCR rate of sandwich neoadjuvant treatment in locally advanced rectal cancer may translate into a better long-term survival benefit: 5-year outcome of a Phase II clinical trial. Cancer Manag Res. 2018;10:4363-4369. doi:10.2147/ CMAR.S168573

6. Garcia-Aguilar J, Chow OS, Smith DD, et al. Effect of adding mFOLFOX6 after neoadjuvant chemoradiation in locally advanced rectal cancer: a multicentre, phase 2 trial. Lancet Oncol. 2015;16 (8):957-966. doi:10.1016/S1470-2045(15)00004-2

7. Bujko K, Wyrwicz L, Rutkowski A, et al. Long-course oxaliplatin-based preoperative chemoradiation versus $5 \times 5$ Gy and consolidation chemotherapy for cT4 or fixed cT3 rectal cancer: results of a randomized Phase III study. Ann Oncol. 2016;27 (5):834-842. doi:10.1093/annonc/mdw062

8. Gollub MJ, Blazic I, Bates DDB, et al. Pelvic MRI after induction chemotherapy and before long-course chemoradiation therapy for rectal cancer: what are the imaging findings? Eur Radiol. 2019;29 (4):1733-1742. doi:10.1007/s00330-018-5726-2

9. Petrillo A, Fusco R, Granata V, et al. Assessing response to neo-adjuvant therapy in locally advanced rectal cancer using intra-voxel incoherent motion modelling by DWI data and standardized index of shape from DCE-MRI. Ther Adv Med Oncol. 2018;10:1758835918809875. doi:10.1177/1758835918809875

10. Kim S, Han K, Seo N, et al. T2-weighted signal intensity-selected volumetry for prediction of pathological complete response after preoperative chemoradiotherapy in locally advanced rectal cancer. Eur Radiol. 2018;28(12):5231-5240. doi:10.1007/s00330-0185520-1

11. Palmisano A, Esposito A, Di Chiara A, et al. Could early tumour volume changes assessed on morphological MRI predict the response to chemoradiation therapy in locally-advanced rectal cancer? Clin Radiol. 2018;73(6):555-563. doi:10.1016/j.crad.2018.01.007

12. Lee ES, Kim MJ, Park SC, et al. Magnetic resonance imaging-detected extramural venous invasion in rectal cancer before and after preoperative chemoradiotherapy: diagnostic performance and prognostic significance. Eur Radiol. 2018;28(2):496-505. doi:10.1007/s00330-017-4978-6

13. Horvat N, Carlos Tavares Rocha C, Clemente Oliveira B, Petkovska I, Gollub MJ. MRI of rectal cancer: tumor staging, imaging techniques, and management. Radiographics. 2019;39 (2):367-387. doi:10.1148/rg.2019180114

14. Smith NJ, Barbachano Y, Norman AR, Swift RI, Abulafi AM, Brown G. Prognostic significance of magnetic resonance imaging-detected extramural vascular invasion in rectal cancer. $\mathrm{Br}$ J Surg. 2008;95(2):229-236. doi:10.1002/bjs.5917
15. Chand M, Swift RI, Tekkis PP, Chau I, Brown G. Extramural venous invasion is a potential imaging predictive biomarker of neoadjuvant treatment in rectal cancer. $B r J$ Cancer. 2014;110(1):19-25. doi:10.1038/bjc.2013.603

16. Li J, Wang J, Pang J, Cao S, Chen J, Xu W. Optimized parameters of diffusion-weighted MRI for prediction of the response to neoadjuvant chemoradiotherapy for locally advanced rectal cancer. Biomed Res Int. 2019;2019:9392747. doi:10.1155/2019/9392747

17. Liu Z, Zhang XY, Shi YJ, et al. Radiomics analysis for evaluation of pathological complete response to neoadjuvant chemoradiotherapy in locally advanced rectal cancer. Clin Cancer Res. 2017;23 (23):7253-7262. doi:10.1158/1078-0432.CCR-17-1038

18. Xiao J, Tan Y, Li W, et al. Tumor volume reduction rate is superior to RECIST for predicting the pathological response of rectal cancer treated with neoadjuvant chemoradiation: results from a prospective study. Oncol Lett. 2015;9(6):2680-2686. doi:10.3892/ol.2015.3101

19. Ryan R, Gibbons D, Hyland JM, et al. Pathological response following long-course neoadjuvant chemoradiotherapy for locally advanced rectal cancer. Histopathology. 2005;47(2):141-146. doi:10.1111/ j.1365-2559.2005.02176.x

20. Phongkitkarun S, Tohmad U, Larbcharoensub N, Sumbunnanondha K, Swangsilpa T, Sirachainan E. DCE-MRI-derived parameters as predictors of response to neo-adjuvant chemoradiation treatment of rectal carcinoma. J Med Assoc Thai. 2016;99(3):338-347.

21. Bakke KM, Hole KH, Dueland S, et al. Diffusion-weighted magnetic resonance imaging of rectal cancer: tumour volume and perfusion fraction predict chemoradiotherapy response and survival. Acta Oncol. 2017;56(6):813-818. doi:10.1080/0284186X.2017.1287951

22. Nagtegaal ID, Glynne-Jones R. How to measure tumour response in rectal cancer? An explanation of discrepancies and suggestions for improvement. Cancer Treat Rev. 2020;84:101964. doi:10.1016/j. ctrv.2020.101964

23. Perez K, Safran H, Sikov W, et al. Complete neoadjuvant treatment for rectal cancer: the Brown University oncology group CONTRE Study. Am J Clin Oncol. 2017;40(3):283-287. doi:10.1097/ COC.0000000000000149

24. Gao YH, An X, Sun WJ, et al. Evaluation of capecitabine and oxaliplatin administered prior to and then concomitant to radiotherapy in high risk locally advanced rectal cancer. $J$ Surg Oncol. 2014;109(5):478-482. doi:10.1002/jso.23516

25. Marco MR, Zhou L, Patil S, et al. Consolidation mFOLFOX6 chemotherapy after chemoradiotherapy improves survival in patients with locally advanced rectal cancer: final results of a multicenter Phase II Trial. Dis Colon Rectum. 2018;61(10):1146-1155. doi:10.1097/DCR.0000000000001207

26. Martens MH, van Heeswijk MM, van den Broek JJ, et al. Prospective, multicenter validation study of magnetic resonance volumetry for response assessment after preoperative chemoradiation in rectal cancer: can the results in the literature be reproduced? Int J Radiat Oncol Biol Phys. 2015;93(5):1005-1014. doi:10.1016/j. ijrobp.2015.09.008

27. Zhang C, Ye F, Liu Y, Ouyang H, Zhao X, Zhang H. Morphologic predictors of pathological complete response to neoadjuvant chemoradiotherapy in locally advanced rectal cancer. Oncotarget. 2018;9 (4):4862-4874. doi:10.18632/oncotarget.23419

28. Ren DL, Li J, Yu HC, et al. Nomograms for predicting pathological response to neoadjuvant treatments in patients with rectal cancer. World J Gastroenterol. 2019;25(1):118-137. doi:10.3748/wjg.v25.i1.118

29. Patel UB, Brown G, Machado I, et al. MRI assessment and outcomes in patients receiving neoadjuvant chemotherapy only for primary rectal cancer: long-term results from the GEMCAD 0801 trial. Ann Oncol. 2017;28(2):344-353. doi:10.1093/annonc/mdw616

30. Meng Y, Wan L, Zhang C, et al. The predictive value of pre-/postneoadjuvant chemoradiotherapy MRI characteristics for patient outcomes in locally advanced rectal cancer. Acad Radiol. 2019. 
31. Bustamante-Lopez LA, Nahas CSR, Nahas SC, et al. Pathologic complete response implies a fewer number of lymph nodes in specimen of rectal cancer patients treated by neoadjuvant therapy and total mesorectal excision. Int J Surg. 2018;56:283-287. doi:10.1016/j.ijsu.2018.07.001

32. Schurink NW, Lambregts DMJ, Beets-Tan RGH. Diffusion-weighted imaging in rectal cancer: current applications and future perspectives. Br J Radiol. 2019;92(1096):20180655. doi:10.1259/bjr.20180655

33. Chen YG, Chen MQ, Guo YY, Li SC, Wu JX, Xu BH. Apparent diffusion coefficient predicts pathology complete response of rectal cancer treated with neoadjuvant chemoradiotherapy. PLoS One. 2016;11(4):e0153944. doi:10.1371/journal.pone.0153944

34. Blazic IM, Lilic GB, Gajic MM. Quantitative assessment of rectal cancer response to neoadjuvant combined chemotherapy and radiation therapy: comparison of three methods of positioning region of interest for ADC measurements at diffusion-weighted MR Imaging. Radiology. 2017;282(2):418-428. doi:10.1148/radiol.2016151908

35. Bassaneze T, Goncalves JE, Faria JF, Palma RT, Waisberg J. Quantitative aspects of diffusion-weighted magnetic resonance imaging in rectal cancer response to neoadjuvant therapy. Radiol Oncol. 2017;51(3):270-276. doi:10.1515/raon-2017-0025

36. Lovinfosse P, Polus M, Van Daele D, et al. FDG PET/CT radiomics for predicting the outcome of locally advanced rectal cancer. Eur J Nucl Med Mol Imaging. 2018;45(3):365-375. doi:10.1007/s00259-017-3855-5
37. Nie K, Shi L, Chen Q, et al. Rectal cancer: assessment of neoadjuvant chemoradiation outcome based on radiomics of multiparametric MRI. Clin Cancer Res. 2016;22(21):5256-5264. doi:10.1158/1078-0432. CCR-15-2997

38. Jeon SH, Song C, Chie EK, et al. Delta-radiomics signature predicts treatment outcomes after preoperative chemoradiotherapy and surgery in rectal cancer. Radiat Oncol. 2019;14(1):43. doi:10.1186/ s13014-019-1246-8

39. Bibault J-E, Giraud P, Housset M, et al. Deep learning and radiomics predict complete response after neo-adjuvant chemoradiation for locally advanced rectal cancer. Sci Rep. 2018;8:1.

40. Shi L, Zhang Y, Nie K, et al. Machine learning for prediction of chemoradiation therapy response in rectal cancer using pre-treatment and mid-radiation multi-parametric MRI. Magn Reson Imaging. 2019;61:33-40. doi:10.1016/j.mri.2019.05.003

41. Xu Y, Hosny A, Zeleznik R, et al. Deep learning predicts lung cancer treatment response from serial medical imaging. Clin Cancer Res. 2019;25(11):3266-3275. doi:10.1158/1078-0432.CCR-18-2495

\section{Publish your work in this journal}

Cancer Management and Research is an international, peer-reviewed open access journal focusing on cancer research and the optimal use of preventative and integrated treatment interventions to achieve improved outcomes, enhanced survival and quality of life for the cancer patient.
The manuscript management system is completely online and includes a very quick and fair peer-review system, which is all easy to use. Visit http://www.dovepress.com/testimonials.php to read real quotes from published authors. 